\title{
A PRELIMINARY REPORT UPON THE BACTERIO- LOGICAL EXAMINATION OF THE CORTEX AND CEREBRO-SPINAL FLUID IN FORTY-SEVEN CASES OF INSANITY.
}

By H. A. TOMLINSON, M. D.

Superintendent St. Peter State Hospital, St. Peter, Minn.

This preliminary report of the results obtained from the routine examination of the cortex and cerebro-spinal fluid, bacteriologically, in all cases dying and coming to post-mortem examination in this hospital, is made now because the systematic report which I expected to make has had to be postponed.

These routine examinations were begun in the spring of 1895 , after the appearance of a paper by Prof. H. C. Wood, of the University of Pennsylvania, in which he suggested that the form of mental disturbance known as "acute delirious mania" might in some instances be of bacterial origin. Since that time other similar reports have been made, and in the "Medical Record" for August I, I896, Dr. W. L. Babcock, of the St. Lawrence Hospital at Ogdensburg, N. Y., reports a case of acute delirium in which he found streptococci and pneumococci in the cerebrospinal fluid both before and after death, and he was disposed to attribute the inflammatory condition of the brain to the presence of the bacteria as a specific cause.

Our clinical work in this hospital, and the microscopical study of the changes in the cortex, found after death in cases of acute insanity, in relation with the diseased conditions present in the chest cavity and abdominal viscera, have led us to entirely different conclusions; viz., that the presence of micro-organisms in the cerebro-spinal fluid and cortex involved their pre-existence in some other part of the organism, and that their presence during the course of acute mental disturbance was not relational or causative, but associative. The details of this association of bac- 
teria with the changes found in the brain after death, occurring during the course of acute mental disease, form the subject of a paper which I have in course of preparation, and I will confine myself in this paper to the simple relation of the findings from the routine bacteriological examination of the cerebro-spinal fluid and cortex.

Knowing how easily the results obtained by these examinations might be vitiated by the contamination of the fluid during its removal, and also the liability of the brain itself to contamination from handling, special precautions were taken, and the fact that in a certain number of cases the cerebro-spinal fluid and cortex were found to be sterile would indicate that these precautions had been fairly successful.

Altogether forty-seven examinations were made, and the form of mental disturbance present at the time of death varied from simple excitement or depression to stupor or prolonged terminal dementia. It was thought best to make the cultures in every case, without regard to the presence or absence of active mental disturbance, thus giving the results attained a greater significance than they would otherwise have.

The cases examined were as follows:

Case I. F. A. S. Acute delirium. Cause of death, acute nephritis. Streptococci in the cerebro-spinal fluid.

Case 2. N. S. Acute excitement. Cause of death, typhoid fever. Typhoid or colon bacillus in the cerebro-spinal fluid. The distinction could not be made.

Case 3. A. H. Acute stupor. Cause of death, chronic parenchymatous nephritis. Growth of colon bacillus from the cerebro-spinal fluid.

Case 4. T. H. Paretic dementia. No active mental disturbance. Cause of death, pneumonia. Spirillum of Obermeier found in the cerebro-spinal fluid.

Case 5. R. G. General paralysis. No active mental disturbance. Cause of death, hypostatic pneumonia. Fraenkel's pneumococcus grown from the cerebro-spinal fluid.

Case 6. S. J. Paretic dementia. No active mental disturbance. Cause of death, acute uræmia. Cerebro-spinal fluid sterile.

Case 7. A. O. General paralysis. No active mental disturbance. Cause of death, cerebral hemorrhage. Staphylococcus 
pyogenes aureus and bacillus pyocyaneus grown from the cerebro-spinal fluid.

Case 8. G. K. Senile insanity. No active mental disturbance. Cause of death, chronic interstitial nephritis. Bacillus pyocyaneus grown from the cerebro-spinal fluid.

Case 9. P. L. Senile insanity. No active mental disturbance. Cause of death, chronic interstitial nephritis. Fraenkel's pneumococcus grown from the cerebro-spinal fluid; also stained in section in the pituitary body.

Case 10. H. B. Senile insanity. No active mental disturbance. Cause of death, pulmonary œdema. Cerebro-spinal fluid and cortex sterile.

Case II. M. B. Senile insanity. No active mental disturbance. Cause of death, cerebral embolism. Pure cultures of Fraenkel's pneumococcus from the cortex and cerebro-spinal fluid.

Case 12. A. R. Senile insanity. No active mental disturbance. Cause of death, uræmia. Spirillum of Obermeier found in cerebro-spinal fluid, but it made no growth on culture media.

Case 13. L. N. B. Senile insanity. No active mental disturbance. Cause of death, chronic parenchymatous nephritis. Cerebro-spinal fluid and cortex sterile.

Case 14. A. G. Terminal dementia. No active mental disturbance. Cause of death, chronic parenchymatous nephritis. Cerebro-spinal fluid contained diplococci which retained their color by Gram, but gave no growth on culture media.

Case 15. E. B. Terminal dementia. No active mental disturbance. Cause of death, pulmonary tuberculosis. Tubercle bacillus in lung cavity was stained, but inoculation of cerebrospinal fluid on culture media gave no growth.

Case 16. A. J. Terminal dementia. No active mental disturbance. Cause of death, interstitial nephritis. Cerebro-spinal fluid and cortex sterile.

Case 17. A. T. Terminal dementia. No active mental disturbance. Cause of death, lobar pneumonia. Fraenkel's pneumococcus grown from pericardial fluid, but no bacteria were found in the cortex or cerebro-spinal fluid.

Case 18. C. L. Terminal dementia. No active mental disturbance. Cause of death, pulmonary tuberculosis. Cerebrospinal fluid sterile. 
Case 19. A. J. Terminal dementia. Depressed, marked selfdepreciation. Suicide by hanging. All organs healthy except the kidneys, which showed chronic interstitial change. The cortex and cerebro-spinal fluid were sterile.

Case 20. O. O. Terminal dementia. No active mental disturbance. Cause of death, pulmonary tuberculosis. Smear preparation of lung tissue showed tubercle bacilli, but there was no growth from the cerebro-spinal fluid.

Case 2I. T. M. Terminal dementia. No active mental disturbance. Cause of death, pulmonary tuberculosis. Bacillus tuberculosis stained in fluid from the pleural cavity, but no tubercle bacilli could be found in the cerebro-spinal fluid.

Case 22. W. N. H. Terminal dementia. No active mental disturbance. Cause of death, pulmonary tuberculosis. The colon bacillus and an unknown spirillum were grown from the cerebro-spinal fluid.

Case 23. J. K. Terminal dementia. No active mental disturbance. Cause of death, lobar pneumonia. The staphylococcus pyogenes albus was grown from the cerebro-spinal fluid. The urine contained Fraenkel's pneumococcus.

Case 24. A. D. Terminal dementia. No active mental disturbance. Cause of death, pulmonary tuberculosis. Growth of staphylococcus pyogenes albus from the cerebro-spinal fluid. The tubercle bacillus was present also.

Case 25. W. D. Terminal dementia. No active mental disturbance. Cause of death, cerebral tumor. Growth of blastomycetes from cerebro-spinal fluid.

Case 26. J. C. Terminal dementia. No active mental disturbance. Cause of death, pulmonary tuberculosis. Smear preparations and sections of a tubercle in the cortex of the uncus showed the tubercle bacillus.

Case 27. N. A. Terminal dementia. No active mental disturbance. Cause of death, cerebral syphilis. Blastomycetes were stained in the cerebro-spinal fluid and grown from inoculation of the diseased cortical area. The syphilis bacillus of Van Niessen was also found in this case.

Case 28. O. J. Terminal dementia. No active mental disturbance. Cause of death, pulmonary tuberculosis. Tubercle bacilli in the cerebro-spinal fluid. 
Case 29. R. W. Terminal dementia. Depressed, with suicidal impulse. Suicide by throwing himself under the cars. Growth of staphylococcus pyogenes albus from the cerebro-spinal fluid. (There was no injury to the head in this case.)

Case 3o. R. O. Terminal dementia. No active mental disturbance. Cause of death, cerebral tumor. Growth of syphilis bacillus (Van Niessen's) from cerebro-spinal fluid. The same organism was stained in section in cerebral gummata.

Case 3I. A. R. Terminal dementia. No active mental disturbance. Cause of death, pulmonary tuberculosis. Fraenkel's pneumococcus grown from the cerebro-spinal fluid. Tubercle bacilli were present in the sputum before death and in the lung tissue after death.

Case 32. J. H. Terminal dementia. No active mental disturbance. Cause of death, pulmonary tuberculosis. Growth of staphylococcus pyogenes albus from the cerebro-spinal fluid.

Case 33. M. N. Terminal dementia. No active mental disturbance. Cause of death, sarcomatosis. Blastomycetes were stained in cortical sarcomata and were grown readily from vaginal exudate. No growth from the cerebro-spinal fluid.

Case 34. C. E. Terminal dementia. No active mental disturbance. Cause of death, chronic parenchymatous nephritis. Growth of Fraenkel's pneumococcus from the cerebro-spinal fluid.

Case 35. H. I. Terminal dementia. No active mental disturbance. Cause of death, pulmonary tuberculosis. The cerebrospinal fluid contained Fraenkel's pneumococcus, associated with the bacillus pyocyaneus, and the same bacteria were found in the urine before death.

Case 36. G. P. Terminal dementia. No active mental disturbance. Cause of death, lobar pneumonia. Fraenkel's pneumococcus grown from the cerebro-spinal fluid, and was also found in the pericardial and peritoneal fluid and in lung tissue.

Case 37. K. K. Terminal dementia. No active mental disturbance. Cause of death, typhoid fever. Colon bacillus grown from the cerebro-spinal fluid.

Case 38. W. S. Terminal dementia. Complete stupor for a month before death. Cause of death, lobar pneumonia. The bacillus pyocyaneus and Fraenkel's pneumococcus were grown from the cerebro-spinal fluid. 
Case 39. B. K. Terminal dementia. Depressed, agitated and confused. Cause of death, chronic parenchymatous nephritis, and there was also a purulent peritonitis. The colon bacillus was grown from the cerebro-spinal fluid and was present in the peritoneal pus.

Case 40. A. C. A. Terminal dementia. No active mental disturbance. Cause of death, mammary carcinoma. The bacillus pyocyaneus was grown from the cerebro-spinal fluid, and the same bacillus with saccharomyces neoformans was found in tumor and a pleural abscess.

Case 4I. F. B. Terminal dementia. No active mental disturbance. Cause of death, chronic interstitial nephritis. Fraenkel's pneumococcus grown from the cerebro-spinal fluid.

Case 42. M. B.S. Terminal dementia. No active mental disturbance. Cause of death, lobar pneumonia with pleuritic abscess. Fraenkel's pneumococcus, bacillus pyocyaneus, staphylococci and streptococci were grown from the cerebro-spinal fluid.

Case 43. L. S. Terminal dementia. Chronic excitement. Cause of death, lobar pneumonia. Fraenkel's pneumococcus found in the cerebro-spinal fluid, and the same organism was grown from the gastric contents during life.

Case 44. H. W. Terminal dementia. Noisy and destructive. Cause of death, pleuro-pneumonia. Fraenkel's pneumococcus and the staphylococcus pyogenes aureus were found in the cerebro-spinal fluid, and the latter also in fluid from the pericardial and pleural cavities.

Case 45. A. F. Terminal dementia. No active mental disturbance. Cause of death, chronic interstitial nephritis. Streptococci were grown from the cerebro-spinal fluid and also found in the kidney.

Case 46. J. S. Terminal dementia. Noisy and destructive. Cause of death, uræmia. The bacillus pyocyaneus was found in the cerebro-spinal fluid.

Case 47. S. P. Terminal dementia. No active mental disturbance. Cause of death, pulmonary tuberculosis. The cerebrospinal fluid contained the tubercle bacillus, as did also the pericardial and peritoneal fluids.

By a reference to the foregoing list it will be noted that three of the cases were recent, two of which were acutely excited and the other stupid. In one case where excitement was present, 
streptococci were found, while in one excited case and in one stuporous case the colon bacillus was found.

Four were cases of general paralysis, and in no two of them was the same micro-organism present; yet the mental condition was the same in all of them at the time of death.

There were six cases of senile insanity, manifesting no active form of mental disturbance at the time of death, their condition representing merely different degrees of dementia. In two of these cases the cerebro-spinal fluid was found to be sterile; in two others Fraenkel's pneumococcus was present, while from the other two were grown the bacillus pyocyaneus and the spirillum of Obermeier.

The other thirty-four cases were all of terminal dementia and manifested no active mental disturbance. In seven of these the cerebro-spinal fluid and cortex were sterile; in one an unrecognized diplococcus which stained by Gram was found; while in the balance eight different micro-organisms were found, and in some of the cases two and three different varieties were grown.

Our clinical experience, coupled with the evidence furnished by these cases, would lead to the conclusion that in any case of illness where micro-organisms are present, if the diseased condition is severe or the individual's vitality is low enough to admit of rapid growth and therefore general dissemination of the microorganism present, it will be found in the cerebro-spinal fluid, but why micro-organisms should be present in the cortex in some and absent in other cases we have not been able to determine, nor the fact that sometimes two or more micro-organisms are present in the bodily organs, but only one of them in the cerebro-spinal fluid. The pneumococcus is more commonly found than any other form of micro-organism, and next to this, staphylococci; but a reference to the mental condition of the patients in whom these bacteria are found shows that the presence of these organisms has no significance in relation to the form of mental disturbance, but does bear a more or less constant relation to the bodily disease present.

In those cases in which the cerebro-spinal fluid and cortex were sterile the bodily disease was of short duration or mild. Of the eight cases in which the cerebro-spinal fluid and cortex were sterile, one was a suicide who was physically in gocd condition at the time of death. Three were cases of chronic kidney 
disease where the patient had been in fairly good physical condition up to a short time before death.

Three were cases of pulmonary tuberculosis in whom the disease ran a comparatively short course, and one was a case of pulmonary cedema coming on in an old man soon after a subdural hemorrhage. In those cases in which the pneumococcus (the micro-organism most constantly present in meningitis) was found, thirteen in all, the clinical picture varied widely, but in all the cases some degree of pulmonary congestion was present. In five of the cases there was an acute lobar pneumonia and in another a hypostatic pneumonia. In three the immediate cause of death was nephritis, while in two it was chronic pulmonary tuberculosis, and in one cerebral embolism. In all of these cases the brain changes were those found in terminal dementia, and in none of them was there an acute process present.

These observations will be continued, but in order to make them more definite, a detailed study is being made as follows:

Cultures are now made from the cortex, cerebro-spinal fluid, pleural fluid, pericardial fluid, abdominal fluid, and from the tissue of that organ which is found most diseased at the time of death. As the result of our observations so far, the conclusion seems warranted that deductions concerning the pathology of insanity, based upon the examination of the brain alone, are likely to be fallible.

NotE.-Since writing the above, and in discussion with different bacteriologists concerning the examination of the brain in cases of insanity, I find that examinations of the cerebro-spinal fluid and cortex have been confined to cases presenting symptoms which suggested the presence of bacterial infection, and that no account has been taken of the bodily disease, if any, from which the patient may have been or was still suffering, during the existence of the mental disturbance, as a possible place of origin for the micro-organisms found in the cerebro-spinal fluid. This omission, coupled with the fact that the absence of bacteria in other forms of mental disturbance, has, so far as my reading goes, been inferred and not demonstrated, would at least render doubtful the assumption that any form of acute mental disturbance was bacterial in its origin. Beside cerebral abscess, meningitis, both septic and tubercular, presents the same clinical picture, so far as the form of mental disturbance is concerned, but the form of micro-organism found post mortem is by no means constant.

The examinations made since this report was written, conducted with more elaboration and care, steadily tend to confirm the conclusions expressed in the report. 